Recepción: 18 / 03 / 2018

Aceptación: 29 / 05 / 2018

Publicación: 01 / 08 / 2018

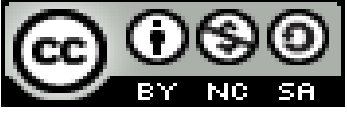

Ciencias de la salud

Artículo de investigación

\title{
Los procesos mentales en la prevención y tratamiento de los niños con atrofia cerebral leve en el manejo integral del aprendizaje
}

\author{
Mental processes in the prevention and treatment of children with mild cerebral \\ atrophy in the integral management of learning
}

Processos mentais na prevenção e tratamento de crianças com atrofia cerebral leve no manejo integral da aprendizagem

\author{
Jeannette M. Coello-Pisco I \\ jannette_cp@outlook.com \\ Adriana G. Viteri- Prieto II \\ adrianaviterip@gmail.com \\ Alba J. Avilés-Salazar III \\ jakita_aviles@hotmail.com
}

Correspondencia: jannette_cp@outlook.com

\footnotetext{
${ }^{\text {I }}$ Magister en Terapia Familiar con Mención en Intervención Sistémica, Licenciada en Ciencias de la Educación Especialidad Supervisión y Administración Educativa, Tecnóloga Medica en Pedagogía Terapéutica, Docente de la Universidad de Guayaquil, Guayaquil, Ecuador.

II Magister en Diseño Curricular, Diploma Superior en Diseño Curricular por Competencias, Profesora en Ciencias de la Educación Especialización Educadores de Párvulos, Licenciada en Ciencias de la Educación Especialización Educadores de Párvulos, Docente de la Universidad de Guayaquil, Guayaquil, Ecuador.

III Diploma Superior en Pedagogía Universitaria, Magister en Educación Superior, Profesora en Ciencias de la Educación Especialización Educadores de Párvulos, Licenciada en Ciencias de la Educación Especialización Educadores de Párvulos, Docente de la Universidad de Guayaquil, Guayaquil, Ecuador.
} 


\section{Resumen}

Este artículo se enfoca en los procesos mentales, en la prevención y tratamiento de los niños con atrofia cerebral leve en el proceso de aprendizaje, esto se refleja en el trabajo clínico de casos presentados al ingreso institucional con un diagnóstico que marca daño a nivel cerebral y un deterioro de las funciones cognitiva. El estudio tiene como objetivo hacer una prevención y tratamiento adecuado en el aprendizaje escolar para poder cumplir un proceso de estrategias pedagógicas en el área cognitiva de los infantes, se estimulará en los procesos mentales que se relacionan con la memoria, atención, concentración, equilibrio, nociones temporales y espaciales, etc. En todas estas capacidades intelectuales se procede a cumplir un proceso de aprendizaje de acuerdo a las necesidades y contenidos de aprendizaje del estudiante, tomando en cuenta el ritmo del aprendizaje. La muestra consiste en 3 pacientes de la Escuela Básica Minerva que pertenece a la Facultad de Filosofía, Letras y Ciencias de la Educación, en la ciudad de Guayaquil. Lo anterior permite analizar casos de cada uno de los niños que presentaron con su diagnóstico médico y que da la oportunidad a canalizar el proceso del aprendizaje para cumplir expectativas o interés para mejorar la calidad de vida de los niños. Así mejora el desempeño de los estudiantes en la enseñanza-aprendizaje, lo que permite aplicar como herramienta o instrumento de la investigación una evaluación con técnicas de entrevista y encuesta previamente valida, cual dio como consecutivamente a lo anterior, se lleva a la aportación de este ponencia principalmente en la parte pedagógica de los niños de inicial 2, en mejorar todo el proceso de aprender para que cada uno pueda ejecutar un aprendizaje significativo, alcance una integración total de los conocimientos y pueda demostrar habilidades y destrezas en el medio que él vive. El mayor pontaje se enfocó en dar una educación integral basado en la parte humanística e integrar equipos multidisciplinarios tanto para el aprendizaje como la salud de los niños del nivel de educación inicial 2. Este equipo multidisciplinario será DECE, médico, directora, representantes legales, docentes y estudiantes, toda una comunidad educativa. Sin olvidar los procesos de seguimiento de una verdadera educación basada en la Neuropedagogía que haga una aportación a todo el trabajo multidisciplinario que se debe hacer para alcanzar logros y estándares de calidad en el aprendizaje de los niños. Los resultados se enfocan en medir cuantitativamente y cualitativamente las necesidades básicas cognitivas de los niños especialmente en una educación integral que beneficiará a toda una comunidad Educativa. La prevención y tratamiento será una reestructura 
escolar a través de programas de inclusión que ayuden a los niños a un tratamiento neuropedagógico y que se realice procesos de estructura clínica y estrategias de aprendizaje en el establecimiento escolar. Esto se acoge a la investigación en la integración educativa, por tal motivo, la comunidad educativa está interesada en trabajar los procesos mentales en el manejo escolar de los niños de la etapa inicial 2, en una forma de educación integral. De esta encuesta los docentes deben conocer de actividades pedagógicas para trabajar con niños con atrofia cerebral leve en la escuela. Por este sentido, es necesario que las actividades pedagógicas se relacionen con los programas de educación especial para orientar todo proceso de aprendizaje. En conclusión, los niños con atrofia cerebral leve, es una función generativa cognitiva, que requiere de un aprendizaje integral cumpliendo normativas y estrategias pedagógicas para alcanzar una educación de calidad. Representa analizar todos los procesos mentales de los niños de 4 a 5 años. La cual no solo imparte conocimientos en el aprendizaje sino en un desarrollo evolutivo integral en la vida de los infantes. El cual evoluciona constantemente y da apertura a tener un aprendizaje significativo y armónico para la vida.

Palabras clave: procesos mentales; funciones cognitivas; proceso aprendizaje; prevención; tratamiento.

\section{Abstract}

This article focuses on the mental processes, prevention and treatment of children with mild cerebral atrophy in the learning process; this is reflected in the clinical work of cases presented at institutional admission with a diagnosis that marks damage at the cerebral level and a deterioration of cognitive functions. The aim of the study is to make a prevention and adequate treatment in school learning to be able to fulfill a process of pedagogical strategies in the cognitive area of infants; it will be stimulated in the mental processes that are related to memory, attention, concentration, balance, temporal and spatial notions, etc. In all these intellectual capacities, a learning process is carried out according to the student's learning needs and contents, taking into account the rhythm of learning. The sample consists of three patients from the Basic Minerva School that belongs to the Faculty of Philosophy, Letters and Education Sciences, in the city of Guayaquil. This allows us to analyze cases of each of the children who presented with their medical diagnosis and who gives the opportunity to channel the learning process to meet expectations or interest to improve the quality of life of children. This improves the performance 
of students in teaching-learning, which allows applying as a tool or instrument of research an evaluation with interview techniques and previously validated survey, which gave as a result of the above, leads to the contribution of this mainly in the pedagogical part of the children of initial 2, to improve the whole process of learning so that each one can execute a meaningful learning, reach a total integration of knowledge and can demonstrate skills and abilities in the environment that he lives. The highest scoreboard focused on providing a comprehensive education based on the humanistic part and integrating multidisciplinary teams for both learning and health of children at the initial education level two. This multidisciplinary team will be DECE, doctor, director, legal representatives, teachers and students, a completely educational community. Without forgetting, the follow-up processes of a true education based on Neuropedagogy that makes a contribution to all the multidisciplinary work that must be done to achieve achievements and quality standards in children's learning. The results focus on quantitatively and qualitatively measuring the basic cognitive needs of children, especially in a comprehensive education that will benefit an entire Educational community. Prevention and treatment will be a school restructuring through inclusion programs that help children to a neuropedagogical treatment and that processes of clinical structure and learning strategies will be carried out in the school setting. This is part of the research into educational integration, for this reason, the educational community is interested in working with the mental processes in the school management of the children of the initial stage 2, in a form of integral education. From this survey, teachers should know about pedagogical activities to work with children with mild cerebral atrophy at school. For this reason, it is necessary that the pedagogical activities be related to the special education programs to guide all learning process. In conclusion, children with mild cerebral atrophy, is a generative cognitive function, which requires a comprehensive learning by complying with rules and pedagogical strategies to achieve a quality education. It represents analyze all the mental processes of children from four to 5 years. Which not only imparts knowledge in learning but in an integral evolutionary development in the life of infants. Which evolves constantly and opens up to have a meaningful and harmonious learning for life.

Keywords: Mental processes; Cognitive functions; Learning process; Prevention; Treatment. 


\section{Resumo}

Este artigo incide sobre os processos mentais na prevenção e no tratamento de crianças com atrofia cerebral processo de aprendizagem leve, isso se reflete no tratamento de casos clínicos apresentados para admissão institucional com um diagnóstico que marcas danificar o cérebro e uma deterioração das funções cognitivas. $\mathrm{O}$ estudo tem como objetivo fazer a prevenção e tratamento adequado na escola aprendendo a cumprir um processo de estratégias de ensino na área cognitiva de bebês, será incentivada nos processos mentais que estão relacionados com a memória, atenção, concentração, equilíbrio, noções temporais e espaciais, etc. Em todas essas capacidades intelectuais, um processo de aprendizado é realizado de acordo com as necessidades e conteúdos de aprendizagem do aluno, levando em consideração o ritmo da aprendizagem. A amostra é composta de três pacientes Minerva Escola Básica pertencentes à Faculdade de Filosofia, Letras e Ciências da Educação, na cidade de Guayaquil. Isso nos permite analisar os casos de cada uma das crianças que tiveram seu diagnóstico médico e dá a oportunidade de canalizar o processo de aprendizagem para atender às expectativas ou juros para melhorar a qualidade de vida das crianças. Isso melhora o desempenho dos alunos no ensino e aprendizagem, que permite que você aplique como uma ferramenta ou instrumento de técnicas de avaliação de pesquisa de entrevista e pesquisa previamente validadas, o que levou consecutivamente ao acima, é preciso a contribuição deste papel ensinando principalmente das crianças inicial dois, melhorar todo o processo de aprendizagem para que todos possam executar uma aprendizagem significativa, alcançar a plena integração de conhecimento e pode demonstrar competências e habilidades no ambiente que ele vive. A maior pontaje focada em fornecer uma educação integral baseada na parte humanista e integrar equipes multidisciplinares para a aprendizagem e para a saúde das crianças de nível de instrução inicial dois. Esta equipe multidisciplinar será DECE, médico, diretor, representantes legais, os professores e estudantes, toda uma comunidade educacional. Sem esquecer os processos de acompanhamento de uma verdadeira educação baseada na Neuropedagogía, que contribui para todo o trabalho multidisciplinar que deve ser feito para alcançar conquistas e padrões de qualidade na aprendizagem das crianças. Os resultados se concentram em medir quantitativa e qualitativamente as necessidades cognitivas básicas das crianças, especialmente em uma educação abrangente que beneficiará toda uma comunidade educacional. Prevenção e tratamento 
será uma reestruturação escolar através de programas de inclusão que ajudem as crianças a um tratamento neuropedagógico e que os processos de estrutura clínica e estratégias de aprendizagem sejam realizados no ambiente escolar. Isso faz parte da pesquisa sobre integração educacional, por isso, a comunidade educativa está interessada em trabalhar com os processos mentais na gestão escolar das crianças do estágio inicial dois, em uma forma de educação integral. A partir dessa pesquisa, os professores devem conhecer as atividades pedagógicas para trabalhar com crianças com atrofia cerebral leve na escola. Por esse motivo, é necessário que as atividades pedagógicas estejam relacionadas aos programas de educação especial para orientar todo o processo de aprendizagem. Em conclusão, crianças com atrofia cerebral leve, é uma função cognitiva generativa, que requer uma aprendizagem abrangente, obedecendo a regras e estratégias pedagógicas para alcançar uma educação de qualidade. Representa analisar todos os processos mentais de crianças de 4 a 5 anos. Que não só transmite conhecimento em aprendizagem, mas em um desenvolvimento evolutivo integral na vida das crianças. Que evolui constantemente e se abre para ter uma aprendizagem significativa e harmoniosa para a vida.

Palavras chave: Processos mentais; Funções cognitivas; Processo de aprendizagem; Prevenção Tratamento.

\section{Introducción}

La presente investigación se refiere al tema de los procesos mentales en la prevención y tratamiento de los niños con atrofia cerebral leve (ACL) en el proceso de aprendizaje. Este trabajo tiene tres enfoques que se relaciona en la parte médica, psicología clínica y educación. Se trabajará en la investigación en el proceso de enseñanza y aprendizaje de los infantes en la importancia del factor escolar. Sin perder los conectores de los otros aspectos que son fortaleza en el proceso de la investigación. La atrofia cerebral leve es un deterioro de las funciones cognitiva de los infantes y se requiere trabajar en los niños a temprana edad especialmente en la parte educativa donde se aplicará la Neuropedagogía. Porque se requiere restaurar los procesos mentales que el niño debe desarrollar a través de las estimulaciones pedagógicas que permite activar las habilidades y destrezas de acuerdo a su edad.

Las características principales de este tipo de dificultad se presentan a nivel de la cabeza. Por esta razón, será mantener activo los conocimientos, saberes y estimular como aprender a desarrollar el pensamiento que está relacionado con las capacidades intelectuales con los procesos mentales o 
cognitivos simples y complejos como la memoria a corto y largo plazo, la atención, equilibrio, nociones temporo espaciales, percepción, lenguaje, inteligencia, pensamiento.

Para analizar esta problemática es necesario mencionar tres causas que están identificada con prenatal, perinatal y postnatal, una de las causas más frecuentes en el momento del parto. Esto hace que el cerebro presente trastornos sensoriales (cognitivo), se da en las dificultades adaptativas en habilidades como la capacidad de aprendizaje, el desarrollar las habilidades y destrezas para adquirir el conocimiento de sí mismo en los diferentes aspectos del saber, social, afectivo y cultural del entorno natural donde se desarrolla el niño. Se debe tomar en cuenta que como afecta principalmente al cerebro dentro de esta área también afecta la parte física y puede mostrar dificultades motrices gruesa y fina. Es necesario, desarrollar al máximo las capacidades mentales de los infantes a temprana edad para canalizar el aprendizaje a su ritmo ya que esta enfermedad es degenerativa y el rol del docente es enfocarse en adaptaciones curriculares (AC), de acuerdo a las necesidades de los niños para mejorar el aprendizaje integral de los niños que tienen esta lección.

Este proceso de investigación busca encontrar posibles soluciones educativas y para esto se aplica instrumentos de investigación a través de la observación directa, encuestas para recoger la información de hecho de acuerdo al contexto de los niños. Para explicar y describir cada proceso que los niños van a desarrollar en el aprendizaje. Esto permitirá limitar el trabajo en la parte educativa.

\section{Importancia del Problema}

Este trabajo de investigación es importante porque acoge como tema de investigación los procesos mentales que están relacionados con la parte cognoscitiva para mejorar la educación de los niños que tienen atrofia cerebral leve en todos los aspectos y que sea una educación integral. La Neuropedagogía ayudará a los procesos de enseñanza y aprendizaje. Prevención en el tratamiento pedagógico de los procesos mentales en los niños con Atrofia Cerebral leve un proyecto de educación inclusiva mediante una investigación de campo en la Escuela Básica Minerva para el diseño de un programa de Adaptación Curricular. Brindar herramientas teóricas y prácticas a toda la comunidad educativa, que los docentes tengan conocimiento del proceso de enseñanza aprendizaje y conozcan técnicas para poder poner énfasis en la creatividad de los niños especiales. 
El docente trabaja desde los saberes para fomentar el hacer cada día a través de la experiencia de aprendizaje de acuerdo a la necesidad de lo que el niño debe aprender de acuerdo a la edad evolutiva y madurativa que se encuentra. Así a través de la experiencia, comienzan a descubrir las habilidades y destrezas que ellos mismo descubren u propia identidad y comparten la responsabilidad propia de ese aprendizaje.

La Neuropedagogía es una rama de las neurociencias que estudia cómo aprende el cerebro. Propone mejorar la calidad del proceso de enseñanza-aprendizaje por medio de la detección de los distintos estilos de aprendizaje para lograr una comunicación eficaz entre docente y estudiante, maximizando el potencial de las competencias cognitivas intelectuales y emocionales de cada educando. Nos permite generar acciones para que los niños sean los protagonistas del proceso de aprendizaje.” (Méndez Adriana, (2015).

Definitivamente, esta estimulación a través del pensamiento y con técnicas apropiadas activa un aprendizaje significativo, porque el docente trabaja con un equipo multidisciplinario donde lo invita al niño a una innovación pedagógica que estimula el aprender para que pueda generar esos cambios en la comprensión y en la enseñanza que requiere aprender. Los procesos mentales serán los primeros estimuladores a través de esa técnica de enseñanza o método que el docente aplique a través de la Adaptación Curricular para el estudiante que ingresa al programa de ayuda especial

Los procesos mentales, corresponden al almacenamiento, elaboración y traducción de los datos aportados por los sentidos, para su utilización inmediata y un eventual uso posterior. La memoria y también la percepción juegan un importante rol entre los procesos cognitivos básicos. La inteligencia, el lenguaje y pensamiento en general son de gran importancia para los seres humanos, ya que forman parte de los procesos cognitivos superiores, ayudándonos a tener procesos mentales que nos diferencian de los animales, como el pensamiento lógico, la utilización de símbolos lingüísticos para transmitir el pensamiento, y la resolución de problemas. Es una operación de pensamiento capaz de actuar sobre estímulos concretos, situaciones o representaciones mentales para generar nuevas representaciones mentales o acciones motoras. (Ramírez Carlos, 2012)

Por ello, se refleja en la mente y en el pensamiento, que cumple un proceso consciente y estructural para desarrollar los nuevos conocimientos a través de la experiencia que vive de 
acuerdo al entorno. También llamada funciones cognitivas que nos permite hacer acciones que ejecuta de acuerdo a su vivir diario y puede almacenar la información que adquiera durante el aprendizaje de su entorno y del mundo. Estas funciones mentales están interconectadas con el aprendizaje escolar la atención, la memoria, las percepciones, razonamiento, equilibrio etc.

En la enseñanza-aprendizaje aparece el Dr. Piaget: Recordemos que son cuatro los estadios que caracterizan el desarrollo cognitivo del niño y del adolescente. El primero se denomina sensoriomotor y abarca el período que va de $\operatorname{los} 0$ a los 2 años, esta etapa es importantísima ya que logra sobre su culminación distintas habilidades motrices y mentales. Los primeros movimientos voluntarios son extensiones de actos reflejos, de allí que la mayoría de sus movimientos se dirigen al propio cuerpo y no a objetos distantes. Promediando este período y ante la creciente coordinación visual motriz, él bebe ya puede dirigir sus actividades a objetos más distantes. En el epilogo de esta fase ya está en condiciones de reprentarse el mundo en imágenes y símbolos mentales, otra característica de esta fase está dada por el inicio del habla que le permite representar objetos ausentes, por último, las actividades lúdicas constituyen un factor muy importante.

El segundo período es el preoperacional, que se extiende desde los 2 años hasta los 7 aproximadamente, asimismo a éste lo podemos dividir en dos subestadios, uno preconceptual que se extiende entre los 2 a 4 años en donde la habilidad más destacada pasa por el razonamiento transductivo, esto significa sencillamente que los niños razonan, pero sin el alcance inductivo ni deductivo, sino yendo de un caso particular a otro caso particular con la finalidad de formar preconceptos, un ejemplo de esto sería cuando los niños observan a sus madres peinándose y en esa ocasión ellas lo hacían para ir de compras, a partir de una situación similar siempre asociarían que salen de compras. Otra particularidad de este período esta signada por el juego simbólico y las conductas egocéntricas. (E. Albornoz Marcelo, 2015).

Por lo tanto, estos periodos son los que representa la parte de Jean Piaget que habla de la parte evolutiva del niño donde da la oportunidad de saber cómo hacer ese proceso de conocimiento y asimilación de acuerdo a su periodo de crecimiento que es necesario aplicar de acuerdo a la edad del niño. Aplicar lo aprendido permite estimular los saberes, pero abre una puerta de la 
experiencia de acuerdo al entorno del niño donde canaliza las habilidades y destrezas que a temprana edad el necesita desarrollar.

La atrofia cerebral es el reflejo objetivo más visible del proceso neurodegenerativo, es un predictor independiente del deterioro cognitivo que padece cerca de la mitad de los pacientes con esa enfermedad. Sin embargo, todavía no existe un método estandarizado para la determinación de ambos parámetros, lo que limita su aplicación concreta en la clínica. A diferencia de los síntomas físicos, la disfunción cognitiva ha sido tradicionalmente postergada en la evaluación de la esclerosis múltiple y se manifiesta, sobre todo, con alteraciones en diversas áreas como: la memoria, el procesamiento de la información, de la atención, la velocidad de procesamiento y de las funciones ejecutivas. (A. Loewy Matías, 2016)

Sin embargo, la atrofia cerebral leve afecta especialmente en las funciones cognitiva de los niños y puede presentarse mayores problema a medida que crece y se desarrollan las diferentes características de la evolución de su crecimiento, por eso es muy importante la prevención de este cuidado tanto en la área de la salud, en la psicología clínica y especialmente en la educación escolar en el niño, durante los primeros años de vida es necesario un cuidado al ritmo de las experiencias para poder canalizar un aprendizaje integral en la vida del paciente. Esta prevención se toma en cuenta de donde fue producida la lección en que parte del cerebro por esta razón se requiere un diagnóstico claro y un constante seguimiento neurológico. Esto invita a trabajar en un grupo multidisciplinario que hoy en día se requiere para dar un servicio de calidad en la salud y educción de los niños.

La atrofia cerebral es un proceso patológico en el que se produce una progresiva muerte y eliminación de las neuronas del cerebro, así como las conexiones neuronales y las estructuras nerviosas. (Gratacós Marcel, 2016). En este sentido, la atrofia cerebral varía dependiendo del daño cerebral y en qué lugar lo ocasiono, especialmente en todas las capacidades intelectuales del niño esto ocurre a temprana edad desde antes del nacimiento y en el momento de formarse el cerebro se presenta la anomalía cerebral que marcara en el resto de su vida. Es necesario que como afecta la parte cognitiva se presenta en los procesos mentales ciertas alteraciones, aunque sean muy leve requiere mejorar en todos los aspectos. 
La educación debe de ser integral y completa. Esto significa que debe de incluir un compendio de aspectos y atender a todas las necesidades y manifestaciones de las personas. La educación integral contempla la totalidad de las funciones humanas, por lo que es un concepto que no puede dejarse de lado. Supone una preocupación en tres campos: el del conocimiento, la conducta y la voluntad. El ámbito del conocimiento hace referencia a la instrucción, abarca los conocimientos que una persona culta debe de adquirir para valerse por sí misma y comprender su entorno. (It. Ana, 2018). La prevención será en este campo solamente pedagógico para fortalecer los nuevos saberes que el estudiante requiere aprender y en cada proceso de aprendizaje que los niños necesitan cuando ingresan por primera vez a un aprendizaje escolar. Es necesario, que el infante haya recibido una estimulación en todos los procesos mentales y en especial el lenguaje y la comunicación para poder activar los canales de comunicación y pueda desarrollar esas habilidades y destrezas. Esto aplica a fortalecer una educación integral de los niños en todos los aspectos tanto cognitivo, social, afectivo, cultural para prevenir cualquier situación que se presente en el contexto de los infantes.

El tratamiento que los niños reciban durante el proceso escolar será interdisciplinario, porque se presentan una serie de manejo escolar que se involucra todo método y técnica en las estrategias metodológicas para que dé resultado en un desarrollo integral en lo social, cognitivo, afectivo, familiar y cultural. Recibiendo apoyo individual y familiar, terapias de aprendizaje, habilidades motoras, coordinación temporo espacial y terapias especiales.

\section{Metodología}

La técnica de instrumento de investigación se relaciona a una encuesta donde se focaliza la problemática presentada especialmente en los procesos mentales y en relación a la prevención y tratamiento de los niños con atrofia cerebral leve, en la parte cognitiva., en niños de inicial 2. El tipo de investigación es cuantitativo, cualitativo y descriptivo porque recoge los hechos en el lugar de contexto que ocurrieron en la escuela Básica Minerva, la población se relaciona en 20 docentes y 20 representantes legales dando una muestra en forma general de 40. Esta encuesta está estructurada con cinco preguntas en forma general y abierta, la misma que refleja en las tablas y gráficos. Se aplicó la encuesta a la comunidad educativa como docentes, representantes legales y autoridad de la Escuela Básica Minerva perteneciente a la Facultad de Filosofía, Letras 
y Ciencias de la Educación de la Universidad de Guayaquil, estas encuestas se realizaron el día 25 de agosto del 2017. Todas estas preguntas se realizaron en la institución educativa mencionada para poder recoger la información como parte de un proceso formativo:

\section{¿Considera que estimular los procesos mentales beneficia a los niños con atrofia cerebral leve?}

Esta pregunta se relaciona con el manejo integral de las diferentes actividades intelectuales que el niño desarrolla durante el aprendizaje.

Gráfico $\mathbf{N}^{\circ}$ 1. Los procesos mentales en el manejo escolar

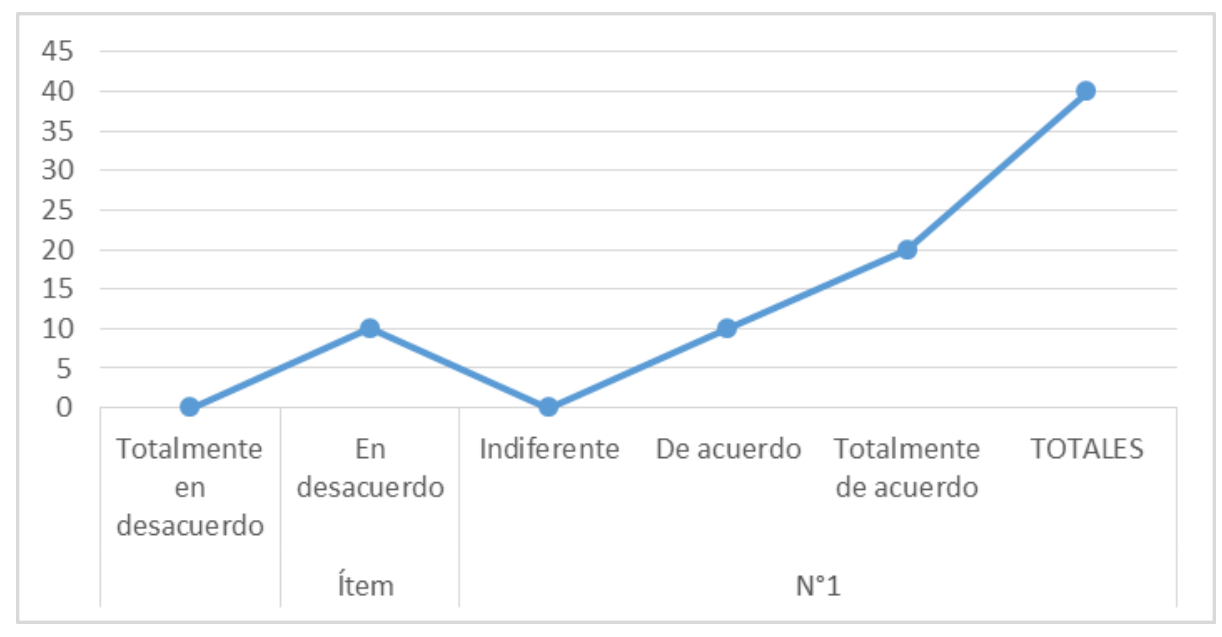

Fuente: Jeannette Coello, Adriana Viteri, Jacqueline Avilés Ecuador 2017

¿Considera que las funciones cognitivas con una adecuada prevención y tratamiento se puede canalizar el aprendizaje integral de los niños que están en la educación inicial dos?

Gráfico $\mathbf{N}^{\circ}$ 2. Educación Integral

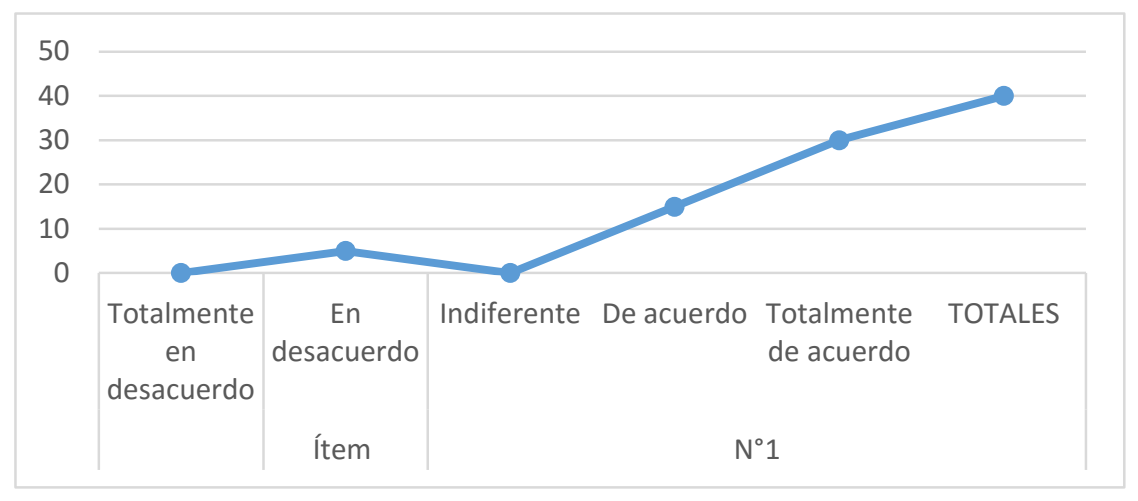

Fuente: Jeannette Coello, Adriana Viteri, Jacqueline Avilés Ecuador 2017 
¿Considera que los docentes deben conocer estrategias de enseñanza de aprendizaje para los niños con atrofia cerebral leve?

Gráfico $\mathbf{N}^{\circ}$ 3. Estrategias de enseñanza- aprendizaje

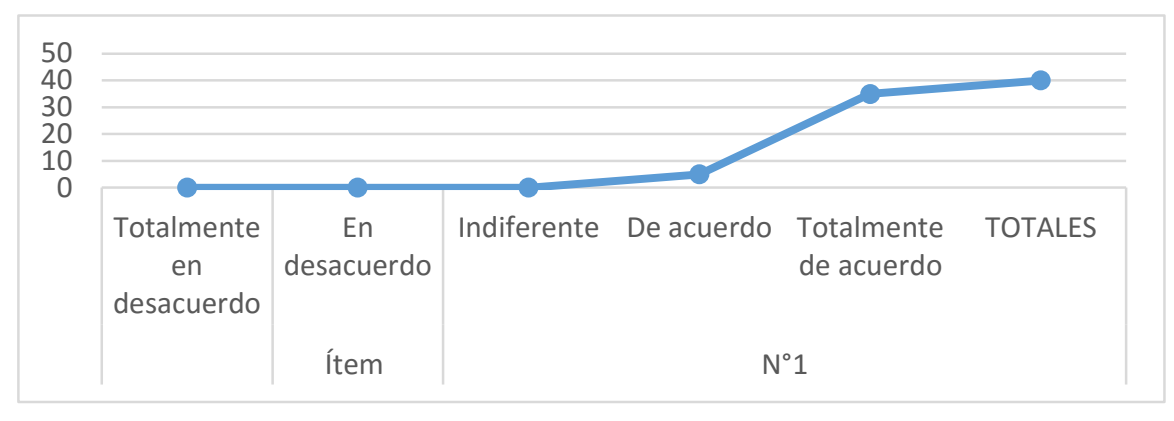

Fuente: Jeannette Coello, Adriana Viteri, Jacqueline Avilés Ecuador 2017

¿Considera que los docentes deben conocer de actividades pedagógicas para trabajar con niños con atrofia cerebral leve en la escuela?

\section{Gráfico $\mathbf{N}^{\circ}$ 4. Actividades pedagógicas}

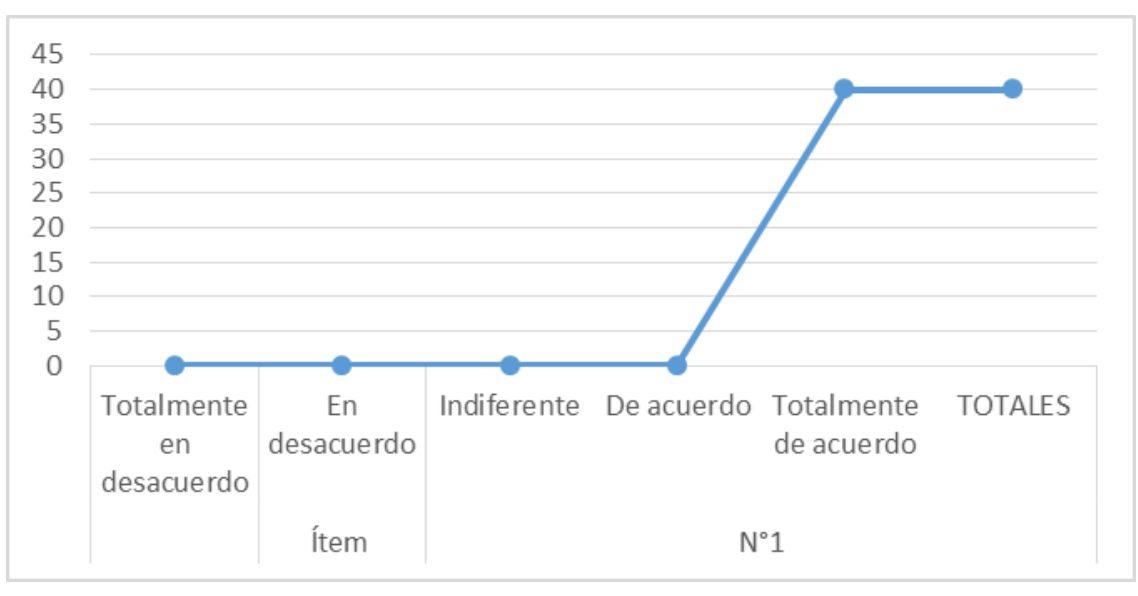

Fuente: Jeannette Coello, Adriana Viteri, Jacqueline Avilés, Ecuador 2017 


\section{¿Cree usted que tener un equipo multidisciplinario en la escuela beneficiara a los niños en} el aprendizaje?

\section{Gráfico $\mathbf{N}^{\circ}$ 5. Equipo multidisciplinario}

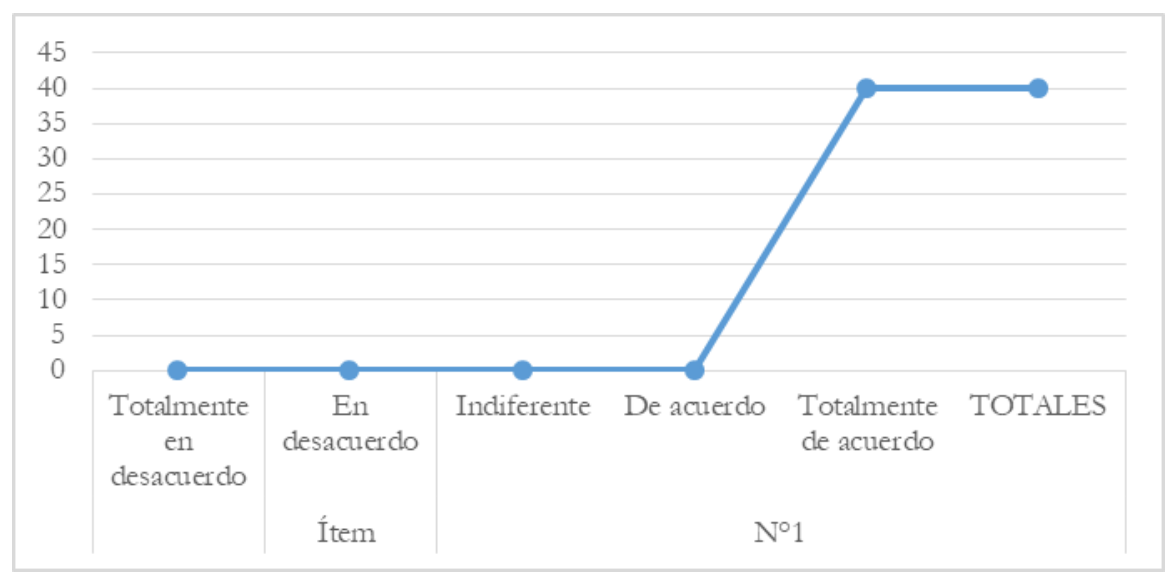

Fuente: Jeannette Coello, Adriana Viteri, Jacqueline Avilés, Ecuador 2017

Este trabajo tiene una relación entre la parte clínica y pedagógica, sin embargo, se trabajará en orientar al docente y al Departamento de Consejería Estudiantil (DECE), para trabajar la parte psicopedagógica y orientar en la enseñanza de aprendizaje integral de los niños. Es importante que estos casos que se presentaron en el 2017 se prestarán para realizar investigaciones, información bibliográfica y poder explicar cómo se orienta con todo un equipo multidisciplinario sumando la pedagogía y la salud mental del futuro niño. Dentro de un marco legal, teórico y basándose en fundamentaciones que dan soporte a la aplicación de las Adaptaciones Curriculares para poder dar una educación de calidad y de acuerdo al ritmo de los niños. El objetivo de esta encuesta es saber cómo se puede mejorar en la parte pedagógica la enseñanza de estos infantes. Es necesario, identificar que esta enfermedad es degenerativa en el área cognitiva por esta razón los programas y contenidos serán de educación especial. Así con el equipo multidisciplinario se podrá lograr una educación integral que complementa la experiencia y los ámbitos de aprendizaje de los niños de la Escuela Básica Minerva.

\section{Resultados}

Los procesos mentales en el manejo escolar manifestaron docentes y representantes legales que de los cuarenta de la muestra veinte de ellos estuvieron Totalmente de acuerdo, diez de acuerdo y 
los otros 10 en desacuerdo. Por tal motivo, la comunidad educativa está interesada en trabajar los procesos mentales en el manejo escolar de los niños de la etapa inicial dos, en una forma de educación integral.

Los docentes y representantes legales en un total de 30 personas, comentaron estar totalmente de acuerdo en las funciones cognitivas con una adecuada prevención y tratamiento que se puede canalizar en el aprendizaje integral de los niños que están en la educación inicial 2. La educación integral cuidará de todos los aspectos del aprendizaje y servirá para fomentar y desarrollar las habilidades y destrezas de los niños de la Escuela Básica Minerva.

De la comunidad educativa 15 manifestaron estar de acuerdo y 5 en desacuerdo. Por ello, es necesario canalizar que la integración sea en todos los aspectos para fortalecer la identidad y autonomía del infante. En el grafico tres, la muestra manifiesta que treinta y cinco de ellos estuvieron totalmente de acuerdo y 5 en desacuerdo. Esto muestra que los docentes deben conocer estrategias de enseñanza de aprendizaje para los niños con atrofia cerebral leve. Por tal motivo, toda estrategia aplicada en el proceso de aprendizaje ayuda a mejorar los procesos de aprendizaje de los niños con estas dificultades cognitivas. De esta encuesta cuarenta de ellos estuvieron de acuerdo en que los docentes deben conocer de actividades pedagógicas para trabajar con niños con atrofia cerebral leve en la escuela. Por este sentido es necesario que las actividades pedagógicas se relacionen con los programas de educación especial para orientar todo proceso de aprendizaje.

Todos en la muestra comentaron estar totalmente de acuerdo en que un equipo multidisciplinario ayude en el proceso pedagógico de la escuela, para beneficiar a los niños del subnivel de inicial 2 que presentan atrofia cerebral leve. Para mejorar las habilidades y destrezas de los niños.

\section{Discusión}

La aportación de este artículo es principalmente en la parte pedagógica de los niños de inicial 2, en mejorar todo el proceso de aprender para que cada uno pueda ejecutar un aprendizaje significativo, para que alcance una integración total de los conocimientos y pueda demostrar habilidades y destrezas en el medio que él vive. El mayor pontaje se enfocó en dar una educación integral basado en la parte humanística e integrar equipos multidisciplinarios tanto para el aprendizaje como la salud de los niños del nivel de educación inicial 2. Este equipo multidisciplinario será DECE, médico, directora, representantes legales, docentes y estudiantes. 
Los docentes deben conocer estrategias de enseñanza de aprendizaje para los niños con atrofia cerebral leve. Por tal motivo, toda estrategia aplicada en el proceso de aprendizaje ayuda a mejorar los nuevos conocimientos de los niños con estas dificultades cognitivas.

\section{Conclusión}

La fase de trabajo que se presenta será de cuidar en todos los aspectos del aprendizaje, cumpliendo normativas y reglamento que hagan una educación de calidad.

Los programas deben estar relacionado con todos los procesos de aprendizaje y especialmente fortalecer un aprendizaje significativo para que los niños alcancen una educación integral.

Toda la comunidad educativa debe fortalecerse de los programas de educación especial para poder adaptar un currículo basado en contenidos, planificaciones que se relacionan de acuerdo a las planificaciones escolares.

Es necesario que ese equipo multidisciplinario debe tener herramientas pedagógicas y recurso didácticos para dar una buena organización de los procesos pedagógicos y en la valoración de valores y comportamientos del niño.

\section{Agradecimiento}

Queremos agradecer a todos los docentes y representantes legales de la Escuela Básica Minerva, que pertenece a la Universidad de Guayaquil, Facultad de Filosofía, Letras y Ciencias de la Educación. Por acogernos en esta investigación del Programa de Inclusión "Abriendo Puertas". Especialmente a la MSc. Adriana Viteri Prieto, directora actual de la institución educativa. Decana de la Facultad de Filosofía Arq. Silvia Moy-Sang Castro, MSc, Vice-decano MSc. Wilson Romero.

\section{Referencias Bibliográficas}

A. Loewy Matías. (23 de noviembre de 2016). Atrofia cerebral: Su papel en las disfunciones cognitivas. Obtenido de https://espanol.medscape.com/verarticulo/5900963

Alfageme González. (23 de noviembre de 2008). Introducción al aprendizaje. Obtenido de Introducción al aprendizaje: capítulo 1. Capítulo 1. Una introducción al aprendizaje... - TDX E. Albornoz Marcelo. (2015). El Aprendizaje según Piaget. Obtenido de https://mayeuticaeducativa.idoneos.com/348494/ 
Gratacós Marcel. (marzo de 4 de 2016). Atrofia Cerebral: Características, Síntomas y Causas. Obtenido de Atrofia Cerebral: Características, Síntomas y Causas: https://www.lifeder.com/atrofia-cerebral/

Guijarro Rosa Blanca. (2016). NUEVA PERSPECTIVA Y VISIÓN. Obtenido de NUEVA PERSPECTIVA $\quad$ Y VISIÓN: http://especial.mineduc.cl/wp content/uploads/sites/31/2016/08/201304151157200.Doc_Nueva_perspectiva_vision_Ed_Especi al.pdf.

It. Ana. (2018) ¿Qué es la educación integral? Obtenido de https://www.cosasdeeducacion.es/que-es-la-educacion-integral/Jimbo. ((2016) p. 196). Neuropedagogía. Obtenido de Neuropedagogía:

Méndez Adriana. (16 de mayo de (2015). ¿Qué es la Neuropedagogía? Obtenido de ¿Qué es la Neuropedagogía?: http://www.neuropedagogia.pe/neuropedagogia-importancia-de-aplicarla-enlos-estudiantes/

Montes de oca Rocío. (2011). Estrategias docentes y métodos de enseñanza-aprendizaje en la Educación Superior. Obtenido de Estrategias docentes y métodos de enseñanza-aprendizaje en la Educación Superior: http://www.humanidadesmedicas.sld.cu/index.php/hm/article/view/127/81

Ramírez Carlos. (2012). procesos mentales. Obtenido de https://planetahola.jimdo.com/psicolog\%C3\%ADa/procesos-mentales/

Sakura. (26 de 0ctubre de 2008). Responsabilidad. Obtenido de Responsabilidad: https://mx.answers.yahoo.com/question/index?qid=20081021141844AAakUQs

Urbano María Auxiliadora. (2016) ¿Cómo lograr integrar los discapacitados auditivos en el estudiantado del liceo Alba Betriz Ramírez? Obtenido de ¿Cómo lograr integrar los discapacitados auditivos en el estudiantado del liceo Alba Betriz Ramírez?: https://issuu.com/mariaurbano9/docs/ma._urbano_-fases. 